\title{
Mechanisms of delayed anti-tuberculosis protection in the lung of parenteral BCG-vaccinated hosts: A critical role of airway luminal $T$ cells
}

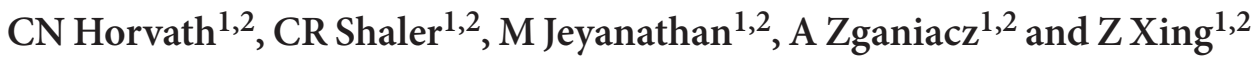

The immune mechanisms underlying unsatisfactory pulmonary mucosal protection by parenteral Bacillus CalmetteGuérin (BCG) immunization remain poorly understood. We found that parenteral BCG immunization failed to elicit airway luminal T cells (ALT) whereas it induced significant T cells in the lung interstitium. After Mycobacterium tuberculosis (M.tb) challenge, ALT remained missing for 10 days. The lack of ALT correlated with lack of lung protection for 14 days post-M.tb challenge. To further investigate the role of ALT, ALT were elicited in BCG-immunized animals by intranasal inoculation of $M$.tb culture-filtrate (CF) proteins. Installment of ALT by CF restored protection in the early phases of $M$.tb infection, which was linked to rapid increases in ALT, but not in lung interstitial T cells. Also, adoptive transfer of $T$ cells to the airway lumen of BCG-immunized animals also accelerated protection. This study thus provides novel evidence that unsatisfactory lung protection by parenteral BCG immunization is due to delayed ALT recruitment after pulmonary M.tb exposure.

\section{INTRODUCTION}

An estimated two billion people worldwide are currently latently infected with Mycobacterium tuberculosis (M.tb) and there are approximately 9.3 million new cases of active tuberculosis (TB) and 1.7 million deaths each year. ${ }^{1}$ The Bacillus CalmetteGuérin (BCG), an attenuated strain of Mycobacterium bovis, is the only approved vaccine against TB and has been administered parenterally via the skin shortly after birth throughout TB endemic areas for nearly a century. ${ }^{2}$ However, while such parenteral BCG vaccination strategy is effective in preventing severe disseminated forms of childhood $\mathrm{TB}$, it has failed to control pulmonary TB, the major cause of the current global TB burden. ${ }^{3-6}$ Despite some progress made in the understanding of the variable efficacy of the $\mathrm{BCG},{ }^{7}$ the precise immune mechanisms underlying the limited capacity of parenteral BCG immunization to protect against TB locally in the lung still remain poorly understood. As parenteral BCG vaccination will continue to be implemented in the human immunization program, enhanced knowledge in this regard will be critical to developing the most effective boosting strategies to enhance the protective immunity in the lung by parenteral BCG priming vaccination.

T-cell-mediated immunity is known to be critical to anti-TB host defense. ${ }^{8}$ As primary TB infection occurs at the respiratory mucosa and it is increasingly recognized that the immunity present at the mucosal site of pathogen entry determines the level of protection, ${ }^{9,10}$ an increased effort is directed towards understanding the kinetics of T-cell recruitment to the lung following pulmonary mycobacterial challenge in naïve or parenterally BCG-vaccinated animals and its relationship with lung protection. ${ }^{8,11,12}$ It has been found that significant T-cell recruitment into the lung of non-vaccinated hosts does not occur until approximately 20 days post-pulmonary myco bacterial challenge. ${ }^{13-16}$ By comparison, a limited number of studies have examined lung $\mathrm{T}$-cell recruitment in parenterally BCG-vaccinated animals and found that vaccination accelerated T-cell recruitment to the lung only by 4-5 days after pulmonary mycobacterial challenge. ${ }^{15,17}$ The general consensus is that although increased lung recruitment of T cells precedes enhanced lung protection, the lung, whether or not with parenteral BCG

${ }^{1}$ McMaster Immunology Research Centre, M.G. DeGroote Institute for Infectious Disease Research, McMaster University, Hamilton, Ontario, Canada. ${ }^{2}$ Department of Pathology and Molecular Medicine, McMaster University, Hamilton, Ontario, Canada. Correspondence: ZXing (xingz@mcmaster.ca) 
vaccination, is still left unprotected in the first 14-20 days after pulmonary mycobacterial challenge. ${ }^{12,18}$ However, of note, all of the studies to date have examined anti-mycobacterial T-cell responses only in the context of the whole lung and it is unclear whether such $\mathrm{T}$ cells are limited to a specific lung compartment. Recent studies on genetic TB vaccines have begun to divide the lung into the two critical tissue compartments-the lung interstitium and airway lumen, when considering T-cell distribution and lung protection. ${ }^{9,12,19}$ A close examination of published studies has revealed that the presence of $\mathrm{T}$ cells in the lung interstitium is not a reliable indicator of lung protection against pulmonary $M . t b$ challenge, whereas those present in the airway luminal space are a more accurate determinant of lung protection. ${ }^{12,20-22}$ Thus, airway luminal $\mathrm{T}$ cells (ALT) are believed to play a critical role in lung mucosal immunity against $M$.tb exposure ${ }^{12,23,24}$ and viral infection. ${ }^{25}$ However, it still remains largely unclear whether antigen (Ag)-specific $\mathrm{T}$ cells populate the airway lumen following parenteral BCG vaccination, how quickly Ag-specific $\mathrm{T}$ cells are recruited into the airway lumen after pulmonary $M$.t $b$ challenge, and what is the relationship between ALT and lung protection in parenteral BCG-vaccinated hosts. Such knowledge will be critical not only to understanding the immune mechanisms of unsatisfactory lung protection by parenteral BCG vaccination but also to developing the effective boosting strategies to enhance protective immunity in the lung by BCG in humans.

In the present study, we have used a murine model of parenteral BCG vaccination to investigate whether parenteral BCG-primed CD4 T cells populate the airway lumen and how quickly these $\mathrm{T}$ cells can be mobilized into the airway lumen upon pulmonary M.tb challenge. Also, we have investigated the role of the ALT in lung protection against pulmonary M.tb infection in these hosts.

\section{RESULTS}

\section{Lack of ALT in parenteral BCG-immunized hosts}

The geographical distribution of T cells plays an important role in mucosal protection from intracellular infections, including TB..$^{9,12,26}$ To examine the tissue distribution of antigen-specific $\mathrm{T}$ cells, particularly CD4 T cells, following parenteral BCG immunization, we immunized mice subcutaneously with BCG and examined Ag-specific CD4 ${ }^{+} \mathrm{IFN}-\gamma^{+}$(interferon- $\gamma$ ) T-cell populations residing in the airway lumen (bronchoalveolar lavage (BAL)), lung interstitium, and spleen 9 weeks post vaccination. There were hardly any detectable Ag-specific CD4 T cells in the airway lumen (BAL; Figure 1a). By contrast, substantial levels of such $\mathrm{T}$ cells were seen in the lung interstitium (lung) and spleen (Figure 1a). These data suggest that parenteral BCG immunization elicits T-cell responses localized to the peripheral tissue sites but not to the airway luminal compartment.

\section{Delayed lung protection and its association with failure of pulmonary M.tb challenge to elicit ALT for up to 10 days in parenteral BCG-immunized hosts}

As the airway lumen was virtually devoid of antigen-specific $\mathrm{T}$ cells following parenteral BCG immunization, we next examined whether and when pulmonary $M . t b$ challenge would be able to elicit Ag-specific airway luminal CD4 T cells (ALT) in parenteral BCG-immunized animals and compared these with the responses in $M . t b \mathrm{H}_{37} \mathrm{Ra}$ challenged non-immunized hosts. We found that up to 14 days post challenge there was a lack of ALT in non-immunized animals (naïve group) (Figure 1b). In comparison, there was still a lack of ALT for at least 10 days post challenge in BCG-immunized animals (BCG group) although the number of ALT was markedly increased by 14 days (Figure 1b). In the lung interstitium of non-immunized animals, the levels of Ag-specific $\mathrm{T}$ cells were very small for at least 14 days post challenge (Figure 1c). In comparison, the levels of such T cells did not markedly rise above BCG-elicited levels until 14 days post challenge (Figure 1c). Thus, after pulmonary M.tb challenge, the overall kinetics of T-cell responses in the lung interstitium of naive or parenteral BCG-immunized animals (Figure 1c) mirror those in the airway lumen (Figure 1b). To examine the relationship between lung T-cell responses and protection, we examined the level of lung immune protection at various time points post-mycobacterial challenge in naive and BCG-immunized animals. We observed that in spite of the presence of Ag-specific $\mathrm{CD} 4 \mathrm{~T}$ cells in the lung interstitium at early times (Figure 1c), the bacterial burden remained comparable in the lungs of naive and BCG-immunized mice for at least 14 days post infection and a marked reduction was not seen until day 28 in BCG-immunized mice (Figure 1d). These results together suggest that (1) parenteral BCG immunization fails to confer any protection in the lung for at least 14 days post challenge; (2), this delayed lung protection is correlated with a complete lack of ALT for up to 10 days of time post challenge in BCG-immunized hosts; and (3) the significant airway luminal T-cell responses (day 14) need to occur before M.tb infection can be markedly reduced in the lung.

\section{Selection of crude M.tb antigenic preparations for recruiting} parenteral BCG-primed T cells into the airway lumen

As parenteral BCG immunization fails to elicit ALT and there is yet still a much delayed appearance of ALT after pulmonary mycobacterial exposure, which is closely linked to the delayed lung protection (Figure 1a-d), we next explored the ways to elicit the ALT in parenteral BCG-immunized mice and compared the efficacies of intranasal (i.n.) inoculation of soluble M.tb antigenic preparations, including recombinant Ag85A (rAg85a), Ag85 complex proteins, and M.tb culture filtrate (CF). To this end, parenteral BCG-immunized mice were exposed to two doses (1 week apart) of selected soluble M.tb Ag and the ability of each Ag to recruit $\mathrm{CD} 4^{+} \mathrm{IFN}-\gamma^{+} \mathrm{T}$ cells into the airway lumen (BAL) and lung interstitium was assessed 4 days later (Figure 2a). Although administration of rAg85a (BCGrAg85a) or Ag85 complex protein (BCG-Ag85 complex) had only a modest effect on eliciting ALT (Figure 2b), exposure to M.tb CF (BCG-immunized/CF-treated (BCG/CF)) exerted a significant increase of ALT ( $P \leq 0.005$; Figure $2 \mathbf{b})$. Similarly, rAg85a or Ag85 complex protein hardly increased the level of lung interstitial $\mathrm{T}$ cells above that by parenteral $\mathrm{BCG}$ immunization alone (Figure 2c). By contrast, the delivery of CF proteins 
significantly increased the number of T cells in the lung interstitium ( $P \leq 0.05$; Figure $2 \mathrm{c})$. CF administrations to naive nonimmunized animals did not induce any detectable Ag-specific T-cell responses in any tissue compartment, indicating that $\mathrm{CF}$ on its own is incapable of T-cell priming (data not shown). As CF delivery was the most effective in eliciting ALT of several tested $M . t b$ antigenic preparations, we carried out histological examination of lung sections and found that compared with BCG, BCG-rAg85a, and BCG-Ag85 complex lungs, BCG/CF

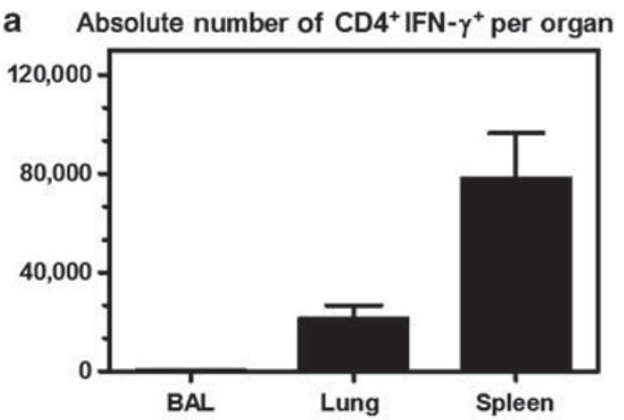

b Absolute number of $\mathrm{CD}^{+} \mathrm{IFN}-\gamma^{+}$per BAL

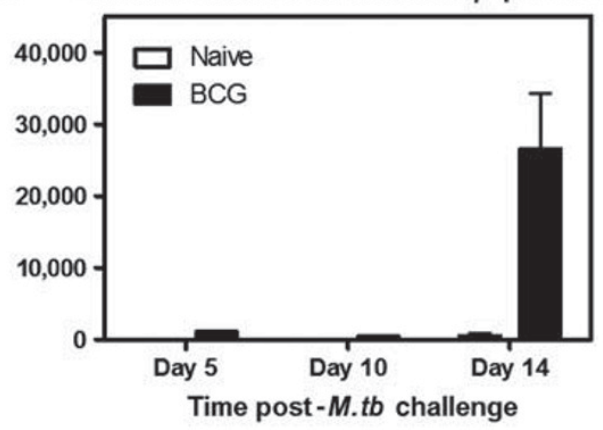

C Absolute number of $\mathrm{CD}^{+} \mathrm{IFN}-\gamma^{+}$per lung

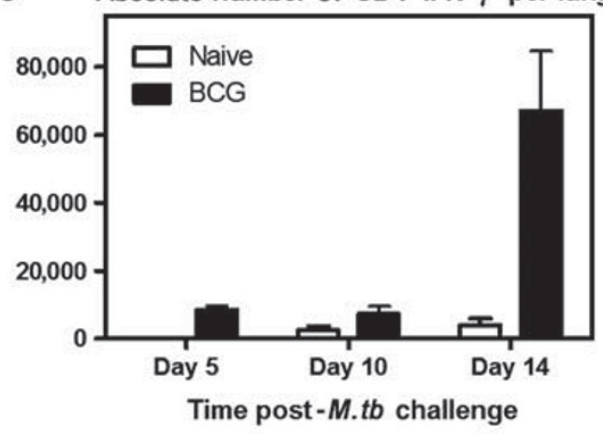

d

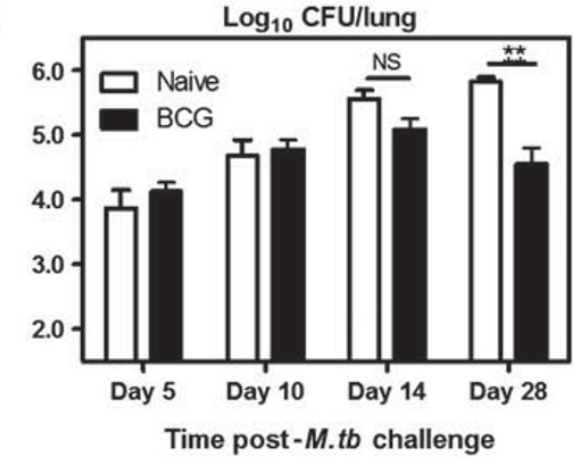

lungs had only a mildly increased cellular infiltration around the airway, in keeping with significantly increased ALT (Supplementary Figure S1A-D online). In addition, the levels of the pro-inflammatory cytokine tumor necrosis factor- $\alpha$ measured in the BAL fluids were also comparable among all the four treatment groups (Supplementary Figure S1E online). Taken together, these data suggest that respiratory mucosal inoculation of soluble CF proteins, but not other mycobacterial Ag preparations, represents an effective and safe way to elicit ALT, which is otherwise missing in parenteral BCG-immunized hosts.

Recruitment of parenteral BCG-primed T cells into the airway lumen by respiratory mucosal inoculation of $\boldsymbol{M}$.tb CF proteins Having identified the i.n. delivery of CF to be the most effective way to elicit ALT in parenteral BCG-immunized animals, we further evaluated its effects in various regimens. First, mice were immunized subcutaneously. with BCG and left for 4 weeks before they were inoculated i.n. with CF, and the Ag-specific CD4 T-cell responses in the airway lumen (BAL) and lung interstitium were examined 2 weeks after the second CF delivery (Figure 3a). Consistent with the data from the previous experiments (Figures 1 and 2), there was a lack of ALT in the lung of parenteral BCG-immunized mice without receiving CF (BCG group; Figure 3b). However, there were a great number of ALT induced by CF treatment $(P \leq 0.005$; BCG/CF group) (Figure $3 \mathbf{b}$ ). On the other hand, as shown in the previous experiments (Figures 1 and 2), parenteral BCG immunization alone elicited a significant level of T cells in the lung interstitium and this level was further increased by i.n. CF delivery $(P \leq 0.0005$; Figure 3c). Based on these findings, we next examined the effect of CF delivery on ALT in a varied immunization regimen whereby the interval between BCG immunization and CF treatment was prolonged from 4 weeks (Figure 3a) to 8 weeks (Figure 3d). We observed that even with an interval of 8 weeks, i.n. CF treatment elicited a significant level of ALT in contrast to a complete lack of ALT in the lung of mice with BCG immunization alone $(P \leq 0.005$; Figure $3 \mathbf{e})$. Similarly, the initially BCG-elevated levels of lung interstitial T cells were further enhanced by CF treatment $(P \leq 0.0005$; Figure 3f).

Figure 1 Lack of airway luminal antigen (Ag)-specific T cells in parenteral Bacillus Calmette-Guérin (BCG)-immunized animals before and after pulmonary mycobacterial challenge. (a) Mice were killed 9 weeks following BCG immunization and Ag-specific T cells in the bronchoalveolar lavage (BAL), lung interstitium, and spleen were examined. (b-d) Naïve non-immunized and BCG-immunized mice were challenged with Mycobacterium tuberculosis (M.tb) 9 weeks after BCG immunization, and killed at 5,10 , and 14 days post infection for T-cell analysis or at $5,10,14$, and 28 days post infection for assessing the level of protection (infection) in the lung. Ag-specific T-cell responses were analyzed in the BAL (b) and lung interstitium (c). Lungs from infected mice were assessed for the level of protection (infection) at various time points post challenge (d). Data are expressed as mean values \pm s.e.m. of 4-5 mice/time/group, representative of 2-3 independent experiments. ${ }^{* \star} P \leq 0.005$ compared with the indicated groups. IFN, interferon; CFU, colony-forming units. 

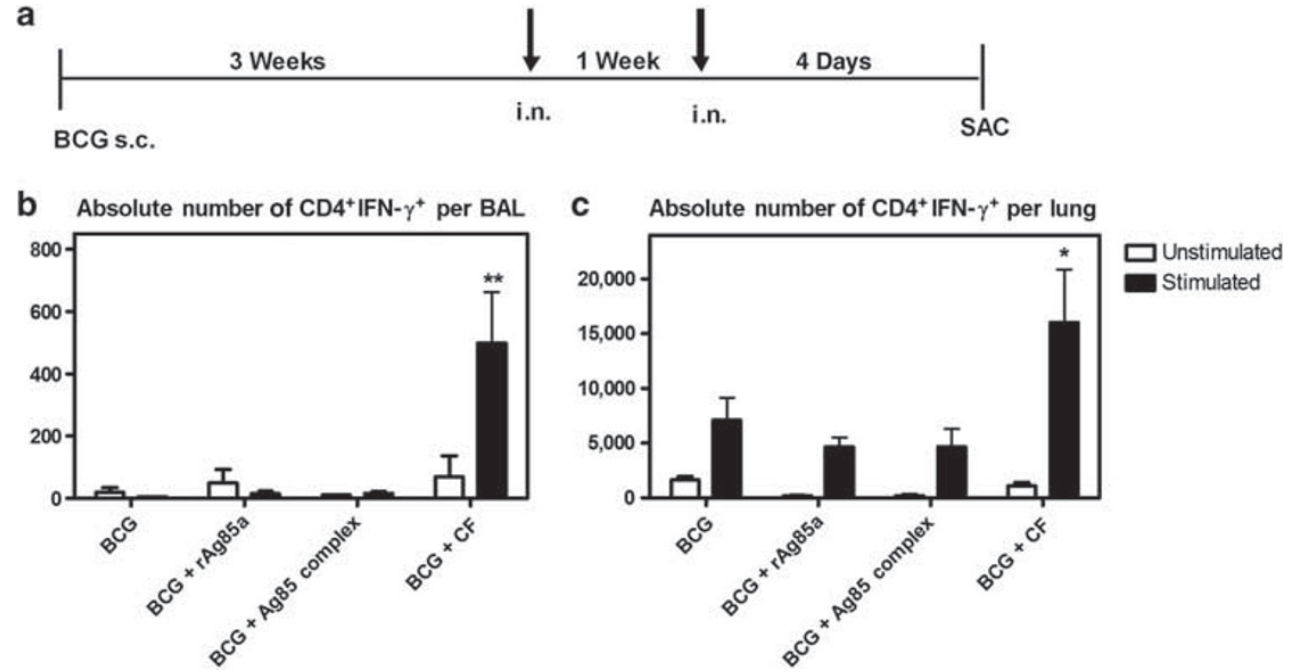

Figure 2 Selection of crude Mycobacterium tuberculosis (M.tb) antigenic preparations for recruiting parenteral Bacillus Calmette-Guérin (BCG)primed T cells into the airway lumen. (a) Experimental schema. (b, c) Four days after the last intranasal (i.n.) delivery of a selected $M$.tb antigenic preparation (recombinant antigen ( $\mathrm{rAg}$ )85A protein, Ag85 complex proteins, or M.tb culture-filtrate (CF) proteins), Ag-specific T cells were analyzed in the bronchoalveolar lavage (BAL) (b) and lung interstitium (c). Data are expressed as mean values \pm s.e.m. of $3-4$ mice per group. ${ }^{*} P \leq 0.05$, ${ }^{\star *} P \leq 0.005$ compared with all the other groups. IFN, interferon; SAC, sacrifice; s.c., subcutaneously.

We next addressed whether the ALT elicited by CF delivery to parenteral BCG-immunized hosts according to the regimen depicted in Figure 3a could persist beyond 2 weeks of time. Thus, mice were set up as in Figure 3a except that the Ag-specific CD4 T-cell responses in the airway lumen (BAL) and lung interstitium were examined 4 weeks after the second CF delivery (Figure 4a). Indeed, compared with the BCG immunization alone group, we detected sharply raised levels of ALT in CF-treated BCG-immunized animals ( $P \leq 0.05$; Figure $4 \mathbf{b}$ ). By comparison, the number of lung interstitial $\mathrm{T}$ cells in the BCG/CF group did not statistically significantly rise above that in the BCG immunization alone group (Figure 4c). The above data together suggest that as long as parenteral BCG-primed $\mathrm{T}$ cells are present within the periphery, they can be effectively mobilized into the airway lumen by airway CF exposure.

\section{Activated effector memory phenotype of ALT elicited by CF in parenteral BCG-immunized hosts}

We next evaluated the immune phenotype and property of ALT elicited by CF in parenteral BCG-immunized hosts. To characterize the activation-memory status of these ALT, we gated on $\mathrm{CD} 4+$ and $\mathrm{CD} 44+\mathrm{T}$ cells and then analyzed the expression of surface markers CD62 L and CD127 on these cells. These surface markers are commonly used for analysis of T-cell phenotypes in the lung. ${ }^{25,26}$ Most of the ALT were found to be CD62 L-CD127 - or CD127 + T cells (Figure 5a), suggesting an effector memory and recently activated effector memory phenotype. The $\mathrm{T}$ cells in the lung interstitium assumed predominantly an effector memory phenotype, being mostly CD62 L-CD127+ (Figure 5b). These data indicate that the ALT elicited by CF treatment in parenteral BCG-immunized hosts are primarily of an activated effector memory phenotype.
The installment of ALT by CF delivery is associated with accelerated and enhanced protection against pulmonary M.tb challenge in parenteral BCG-immunized hosts

To examine whether the reinstated ALT in CF-treated parenteral BCG-immunized animals would confer lung protection that was otherwise missing for up to 14 days post-pulmonary mycobacterial challenge in BCG-immunized hosts (Figure 1d), naïve non-immunized (naive), BCG-immunized (BCG), and BCG/CF mice were challenged via the airway with virulent $M . t b \mathrm{H}_{37} \mathrm{Rv} 4$ weeks after the last $\mathrm{CF}$ administration and the level of lung protection was assessed at 2 weeks post challenge (Figure 6a). Despite the significant presence of Agspecific T cells within the lung interstitium of BCG-immunized animals at the time of challenge and consistent with the data in Figure 1d, the lungs of BCG mice were not any better protected than the naive controls (Figure 6b). However, a significantly enhanced level of protection was seen in the lung of BCG/CF mice $(P \leq 0.005$; Figure $6 \mathbf{b})$. This suggests that the installment of ALT at the time of pulmonary M.tb exposure is associated with accelerated lung protection that is completely lacking in the early phases of pulmonary $M . t b$ infection in parenteral BCG-immunized hosts.

We next went on to address whether this accelerated lung protection in the early phases of M.tb infection seen in BCG/ $\mathrm{CF}$ animals would continue into the later point of infection. To this end, mice were set up as in Figure $\mathbf{6 b}$ except that the level of lung protection was assessed at 4 weeks post-M.tb challenge (Figure 6a). As expected and also in keeping with the data in Figure 1d, parenteral BCG immunization alone by this time conferred a significantly enhanced level of lung protection compared to naive controls $(P \leq 0.005$; Figure $6 \mathbf{c})$. However, the level of lung protection in BCG/CF animals was significantly further enhanced $(P \leq 0.05)$ over the BCG group (Figure $6 \mathbf{c}$ ). 
a
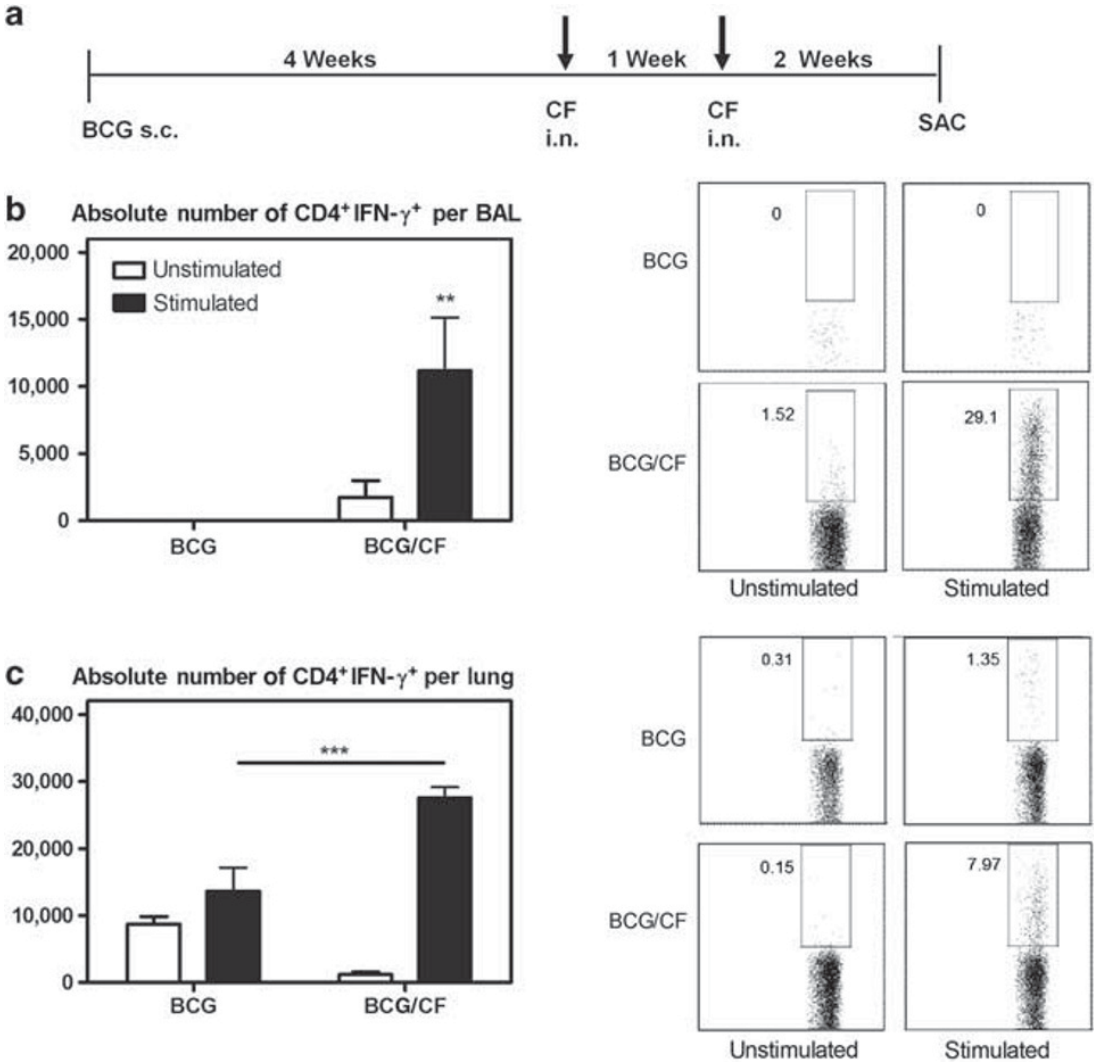

d

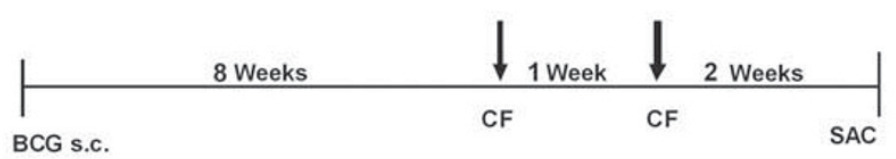

e Absolute number of $\mathrm{CD}^{+} \mathrm{IFN}-\gamma^{+}$per BAL
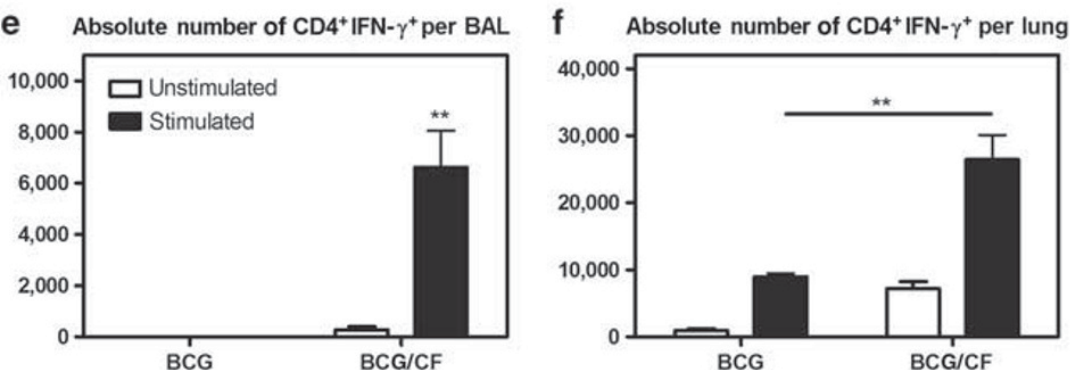

Figure 3 Recruitment of parenteral Bacillus Calmette-Guérin (BCG)-primed T cells into the airway lumen by respiratory mucosal inoculation of crude non-formulated Mycobacterium tuberculosis culture-filtrate (CF) proteins. (a, d) Experimental schema. (b, c) The absolute numbers and representative dot plots of antigen (Ag)-specific T cells in the bronchoalveolar lavage (BAL; b) and lung interstitium (c) analyzed 2 weeks after the second intranasal (i.n.) CF in the mice that received the first CF from 4 weeks post-BCG immunization. (e, f) The absolute numbers and representative dot plots of Ag-specific T cells in the BAL (e) and lung interstitium (f) analyzed 2 weeks after the second i.n. CF in the mice that received the first CF from 8 weeks post-BCG immunization. Data are expressed as mean values \pm s.e.m. of 3-4 mice per group, representative of two independent experiments. ${ }^{* *} P \leq 0.005,{ }^{* * *} P \leq 0.0005$ compared with the indicated groups. IFN, interferon; SAC, sacrifice; s.C., subcutaneously.

There were also correspondingly decreased levels of bacterial burden in the spleen (data not shown). With regard to improved lung protection, the BCG/CF group had much improved lung histopathology compared with the non-immunized naive or BCG immunization groups (Figure $6 \mathbf{e}$ and $\mathbf{f}$ ). The above findings together suggest that airway CF delivery and ALT installment in parenteral BCG-immunized animals accelerate and enhance lung protection against pulmonary M.tb infection.
Highly reactive secondary airway luminal T-cell responses to pulmonary M.tb. challenge in parenteral BCG/CF animals To further understand the mechanisms by which airway CF treatment enhances lung protection in parenteral BCG-immunized hosts, we examined the kinetics of secondary Ag-specific CD4 T-cell responses in the airway lumen following pulmonary mycobacterial challenge. To this end, the mice were set up similarly as depicted in Figure 6a except that T-cell 
a

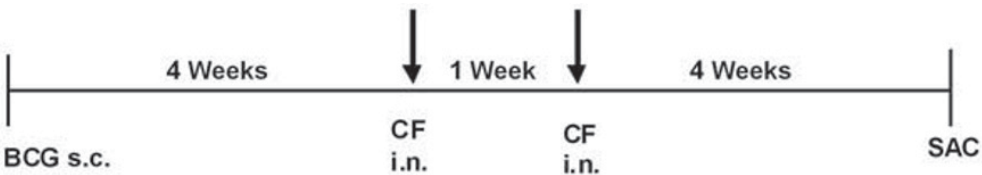

b Absolute number of $\mathrm{CD}^{+}$IFN- $\gamma^{+}$per BAL
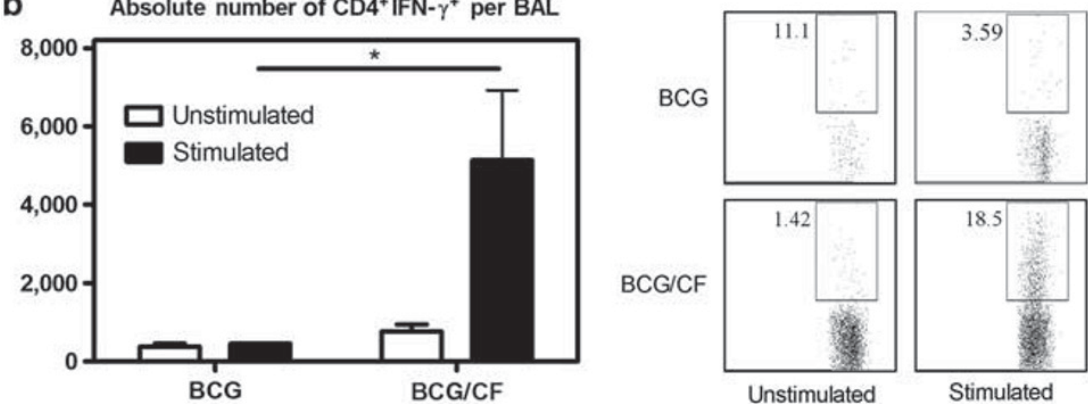

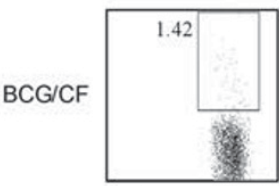

Unstimulated

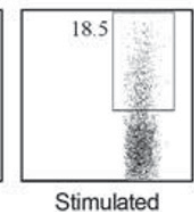

C Absolute number of $\mathrm{CD4}^{+} \mathrm{IFN}-\gamma^{+}$per lung
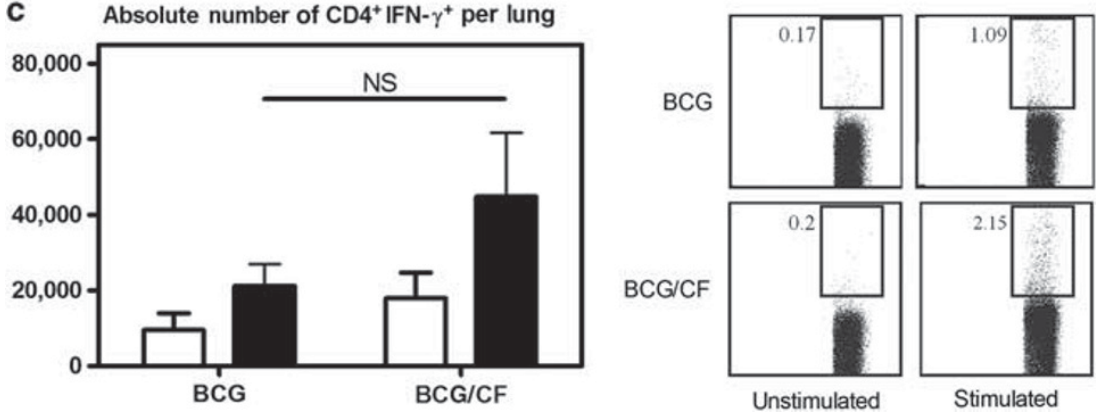

Figure 4 Retained airway luminal T cells by respiratory mucosal inoculation of crude non-formulated Mycobacterium tuberculosis culture-filtrate (CF) proteins in parenteral Bacillus Calmette-Guérin (BCG)-immunized animals. (a) Experimental schema. (b, c) The absolute numbers and representative dot plots of antigen (Ag)-specific T cells in the bronchoalveolar lavage (BAL; b) and lung interstitium (c) analyzed 4 weeks after the second intranasal (i.n.) $\mathrm{CF}$ in the mice that received the first $\mathrm{CF}$ from 4 weeks post-BCG immunization. Data are expressed as mean values \pm s.e.m. of $4-5$ mice per group, representative of four independent experiments. ${ }^{*} P \leq 0.05$ compared with the indicated group. IFN, interferon; NS, not statistically significant; SAC, sacrifice; s.c., subcutaneously.

responses were assessed at $0,5,10$, and 14 days post- $M . t b \mathrm{H}_{37} \mathrm{Ra}$ challenge (Figure 7a). In keeping with the data presented in Figure 1b, there was hardly any detectable ALT in BCG-immunized animals until 14 days post- $M$. $t b$ challenge (Figure $7 \mathbf{b}$ ). In sharp contrast, a raised level of ALT (CD4 T cells) was already present before M.tb exposure in BCG-immunized-CF-treated animals (day 0; Figure 7b) as seen in previous experiments (Figure 4b), and subsequent pulmonary M.tb exposure quickly further increased the levels of ALT several times above the base level at days 5, 10, and 14 (Figure 7b). On the other hand, in keeping with the data in Figure 1a-c, parenteral BCG immunization alone elicited a significant $\mathrm{T}$-cell population in the lung interstitium before pulmonary mycobacterial challenge (day 0 ), and this population did not increase in size until day 14 (Figure 7c; BCG group). In comparison, although BCG/CF animals had a larger $\mathrm{T}$-cell population in the lung interstitium before pulmonary mycobacterial challenge (day 0 ), it remains unchanged between day 5 and day 14 post-mycobacterial challenge (Figure 7c; BCG/CF group), contrasting the highly responsive ALT in these mice (Figure 7b). Similarly, increased Ag-specific CD8 T cells, albeit in smaller numbers than CD4 $\mathrm{T}$ cells, were also seen at various time points in the BCG/CF group following M.tb challenge (Table 1). Thus, the markedly increased secondary T-cell responses soon after pulmonary M.tb exposure in the airway lumen of the BCG immunized/ CF-treated animals group were associated with a significantly increased level of lung protection at day 14 (Figure $\mathbf{6 b}$ and data not shown). These findings suggest that airway CF treatment accelerates and enhances lung protection in parenteral BCGimmunized hosts by installing highly mycobacterium-reactive $\mathrm{T}$ cells in the airway lumen in the early stages of pulmonary M.tb infection, which are otherwise absent in parenteral BCG animals if untreated with CF.

Adoptive transfer of Ag-specific T cells into the airway lumen of parenteral BCG-immunized mice restores lung protection in the early phases of pulmonary M.tb infection

Thus far, we have found that prior respiratory mucosal CF delivery to parenteral BCG-immunized animals accelerates lung protection in the early phases of pulmonary M.tb infection (Figure 6a) and this was associated closely with dramatically increased responses of ALT (Figure 7b), but not with lung interstitial T cells (Figure 7c). To further investigate the role of ALT in lung protection in the early phase of pulmonary M.tb 


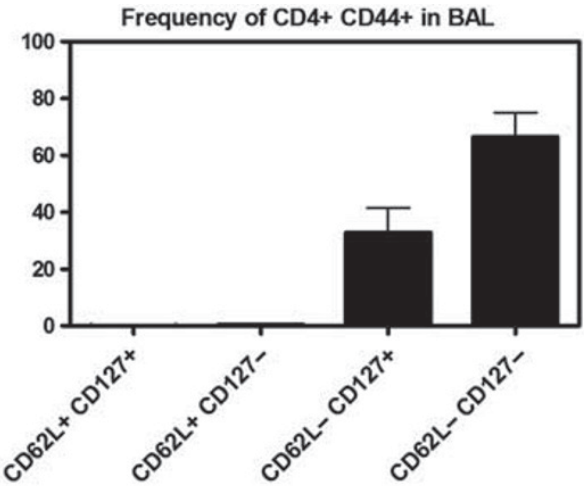

b Absolute number of $\mathrm{CD} 4+\mathrm{CD} 44+$ per lung

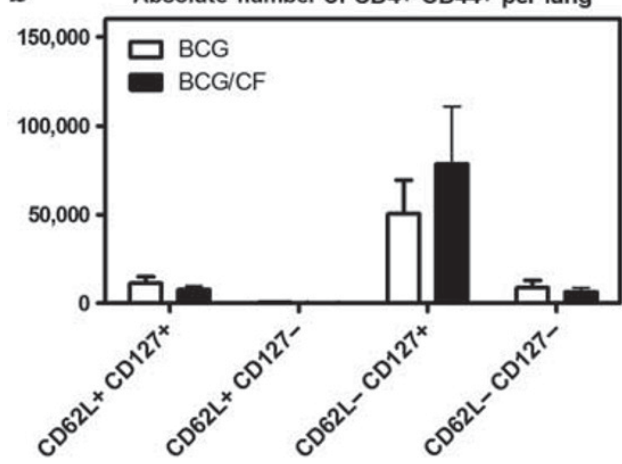

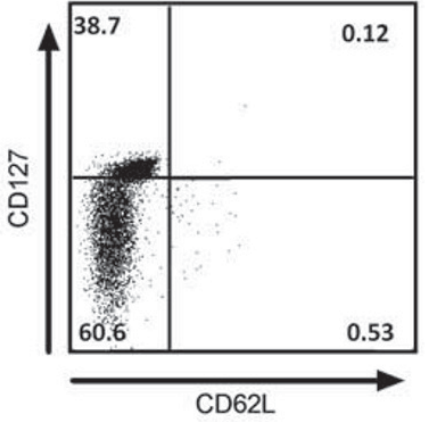

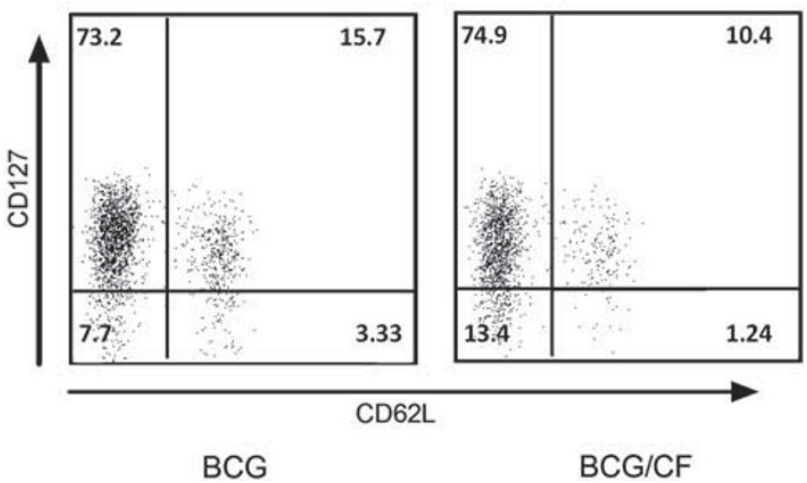

Figure 5 Activated effector memory phenotype of airway luminal T cells elicited by Mycobacterium tuberculosis culture filtrate (CF) in parenteral Bacillus Calmette-Guérin (BCG)-immunized animals. The mice were immunized with BCG for 4 weeks, intranasally inoculated with CF and killed 2 weeks after the second CF as depicted in Figure 3a. The immune phenotype of antigen-specific T cells in the bronchoalveolar lavage (BAL; a) and lung interstitium (b) were analyzed. Data are expressed as mean values \pm s.e.m. or representative dot plots of 4-5 mice per group, representative of three independent experiments.

infection, we addressed whether adoptive transfer of Ag-specific CD4 $\mathrm{T}$ cells to the airway lumen of parenteral BCG-immunized animals (without CF treatment) would accelerate lung protection in the early phase of pulmonary M.tb infection, similarly as did the airway CF delivery. This approach would allow us to specifically examine the role of ALT in early lung protection without altering or increasing the levels of lung interstitial $\mathrm{T}$ cells as we have previously shown that the $\mathrm{T}$ cells adoptively transferred to the airway lumen do not transmigrate to the other sites, including the lung interstitium. ${ }^{26}$ To this end, mice were immunized subcutaneously with BCG for 9 weeks and adoptively transferred intratracheally with purified $\mathrm{T}$ cells isolated from the lungs of BCG/CF mice (BCG-AT (BCG/CF) group) or from the lungs of naive mice as a control (BCG-AT (naïve) group). Additional control groups were naive non-immunized (naive), parenteral BCG alone (BCG), and parenteral BCG/CFtreated $(\mathrm{BCG} / \mathrm{CF})$ mice. The mice were then challenged via the airway with virulent $M . t b \mathrm{H}_{37} \mathrm{Rv}$ on the following day (16 h) after T-cell transfer and killed 2 weeks post challenge for assessment of early lung protection (Figure 8a). As shown in Figures $\mathbf{1 d}$ and $\mathbf{6 b}$, there was a lack of lung protection by day 14 post$M . t b$ challenge in parenteral BCG-immunized mice (naïve vs. BCG groups) (Figure 8b). However, the BCG mice receiving adoptively transferred $\mathrm{Ag}$-primed $\mathrm{T}$ cells isolated from $\mathrm{BCG} / \mathrm{CF}$ mice (BCG-AT (BCG/CF) group) demonstrated a significantly enhanced level of lung protection $(P \leq 0.0005$; Figure 8b), which was comparable with that accomplished in BCG/CF animals (Figure 8b). By contrast, the BCG mice receiving adoptively transferred unprimed $\mathrm{T}$ cells isolated from naive mice (BCGAT (naïve) group) did not have enhanced lung protection (Figure 8b). Collectively, these data further indicate that the Ag-specific ALT is a critical determinant of lung protection in the early phases of pulmonary M.tb infection.

\section{DISCUSSION}

To date, the precise immune mechanisms underlying the unsatisfactory protection against pulmonary TB in BCG-vaccinated individuals remains poorly understood. Our current study reveals that parenteral BCG immunization fails to elicit the ALT although a substantial population of Ag-specific $\mathrm{T}$ cells can be detected within the lung interstitium. The absence of ALT in BCG-immunized animals persists for a significant period of time (at least 10 days) even after pulmonary $M . t b$ challenge and this is well correlated with the complete lack of immune protection in the early phase of pulmonary mycobacterial infection in spite of the presence of lung interstitial T cells. Thus, by recruiting a population of parenterally BCGprimed $\mathrm{T}$ cells into the airway lumen via respiratory mucosal delivery of crude unformulated or unadjuvanted mycobacterial proteins (CF), the lung is granted a significantly enhanced level 

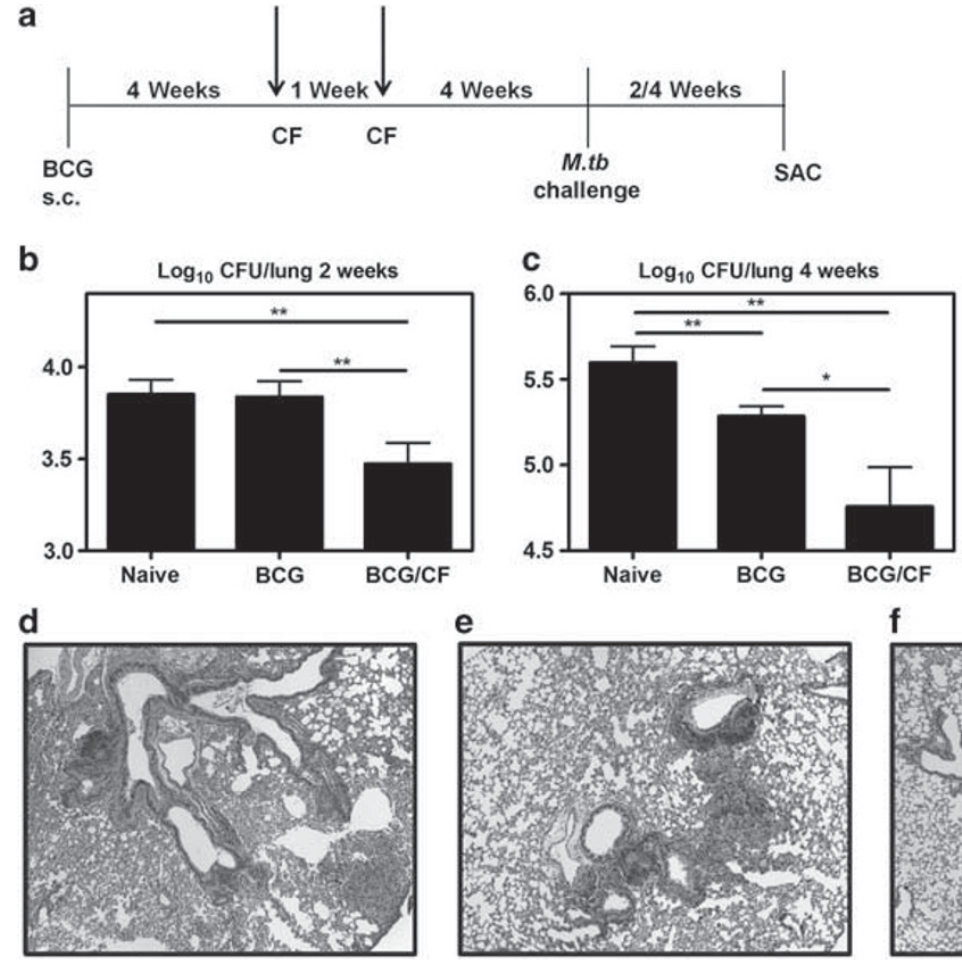

Naive

e

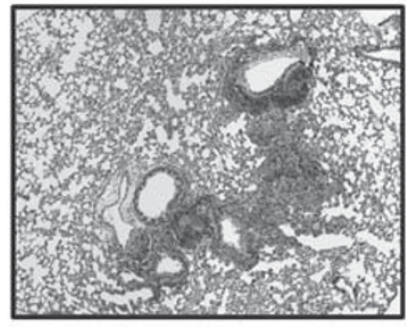

BCG

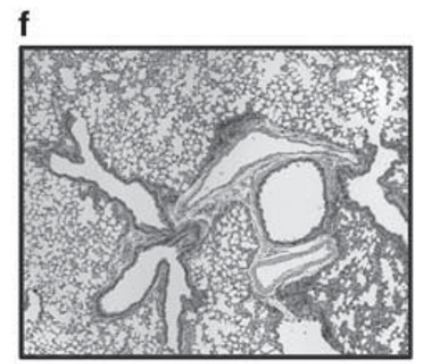

BCG/CF

Figure 6 Accelerated and enhanced immune protection from pulmonary Mycobacterium tuberculosis (M.tb) challenge by culture filtrate (CF)mediated installation of airway luminal T cells in parenteral Bacillus Calmette-Guérin (BCG)-immunized hosts. (a) Experimental schema. (b, c) The levels of immune protection in the lung (bacterial colony-forming units (CFU) burden) were assessed either 2 weeks (b) or 4 weeks (c) post-M.tb challenge. Data are expressed as mean values \pm s.e.m. of 5-6 mice per group, representative of two independent experiments. (d-f) The extent of lung immunohistopathology was assessed at 4 weeks post-M.tb challenge in naïve non-immunized (d), parenteral BCG immunization alone (e) and parenteral BCG-immunized/CF-treated (f) mice. Each hematoxylin and eosin -stained histomicrograph is representative of 5-6 mice per group ( $\times 4$ magnification). ${ }^{\star} P \leq 0.05,{ }^{* *} P \leq 0.005$ compared with the indicated groups. SAC, sacrifice; s.c., subcutaneously.

of immune protection in the early phases of pulmonary M.tb infection. Different from lung interstitial T cells, these initially implanted ALT by mucosal CF delivery are able to undergo a rapid and robust expansion upon mycobacterial exposure. We also demonstrated that the mere equipment of ALT by adoptive airway T-cell transfer, without a change to the size of lung interstitial T-cell population, in parenteral BCG-immunized animals, is able to restore early lung immune protection as effectively as airway CF delivery. These findings together provide new insights into the immune mechanisms behind the delayed and unsatisfactory protection locally in the lung by parenteral BCG immunization, and point to the importance of Ag-specific $\mathrm{T}$ cells present on the surface of respiratory mucosa in immune protection in the early phases of pulmonary M.tb infection.

It has been long believed that the magnitude of immune protection against a mucosal infection is closely associated with the level of immune effectors present at that mucosal site. $9,10,25$ This concept has increasingly been applied to understanding anti-TB immunity and vaccination. ${ }^{8,11,12,18}$ However, although a limited number of studies have begun to address the relationship between the level of immune protection and Ag-specific CD4 $\mathrm{T}$ cells in the lung of naive ${ }^{13-16}$ or parenteral BCG-immunized animals, ${ }^{15,17,27}$ these studies were carried out only by taking the lung as a single entity. In other words, it remains unclear whether the T-cell responses examined in these studies were localized to the lung interstitium, airway lumen, or both. Recent studies on parenteral and mucosal genetic $\mathrm{TB}$ vaccination have suggested the importance of dissecting anti-TB T-cell responses separately on the surface of respiratory mucosa (ALT) and in the lung interstitium. This is because different from the ALT, the mere presence of such $\mathrm{T}$ cells within the lung interstitium may not well inform about the level of anti-TB protection in the lung. ${ }^{12,20-22}$ In addition to genetic vaccines, it is believed that the ALT also hold the key to superior lung protection by respiratory mucosal immunization with other forms of TB vaccines. ${ }^{23,24,28}$ Notably, different from parenteral genetic TB vaccination, parenteral BCG immunization does provide a significant level of lung protection in the later stage of pulmonary M.tb infection as shown in the current study in addition to many other studies. However, we have found that parenteral BCG immunization is unable to protect the lung in the early phases of pulmonary M.tb infection (up to at least 14 days) in spite of a significant presence of lung interstitial Ag-specific CD4 T cells. The delayed immune protection in the lung of these animals coincides with the lack of ALT, whereas the increased protection in later 
a

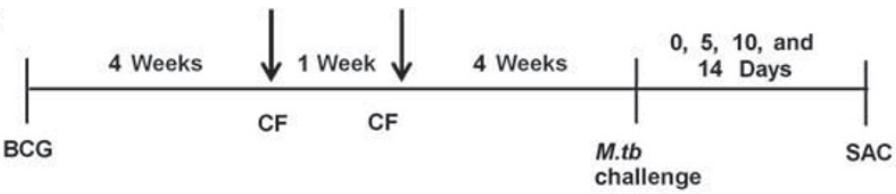

b Absolute number of $\mathrm{CD4}^{+} \mathrm{IFN}-\gamma^{+}$per BAL

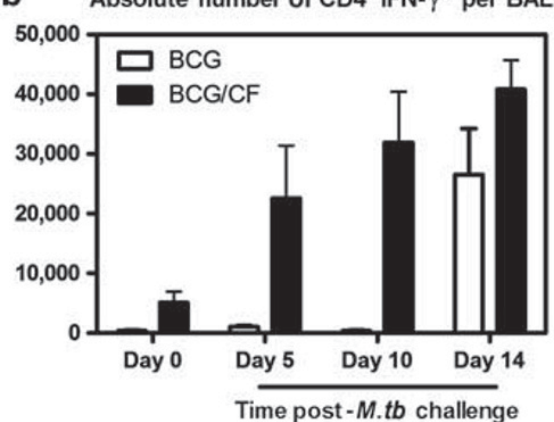

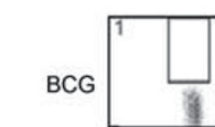
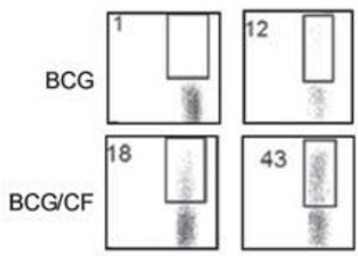

Day 0

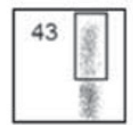

Day 5
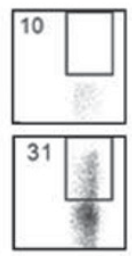

Day 10
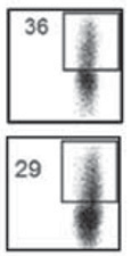

Day 14

C Absolute number of $\mathrm{CD}^{+}$IFN- $\gamma^{+}$per lung

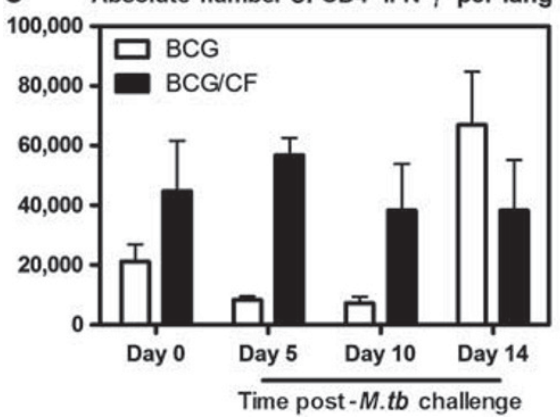

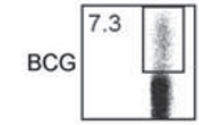

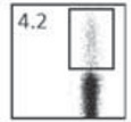

$\mathrm{BCG} / \mathrm{CF}$

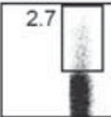

Day 0

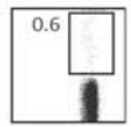

Day 5
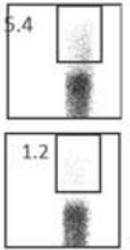

Day 10

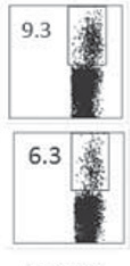

Day 14

Figure 7 Highly reactive secondary airway luminal T-cell responses to pulmonary Mycobacterium tuberculosis (M.tb) challenge in parenteral Bacillus Calmette-Guérin (BCG)-immunized/CF (culture filtrate)-treated hosts. (a) Experimental schema. (b, c) The absolute numbers and representative dot plots of antigen-specific T cells in the bronchoalveolar lavage (BAL; b) and lung interstitium (c) analyzed at various time points post-M.tb challenge in the mice immunized with BCG and not treated with $C F$ (BCG) or immunized with BCG and treated with CF (BCG/CF). Data are expressed as mean values \pm s.e.m. of 4-5 mice per group, representative of two independent experiments. IFN, interferon; SAC, sacrifice.

Table $1 \mathrm{CD}^{+}{ }^{+} \mathrm{IFN}-\gamma^{+} \mathrm{T}$ cells in the BAL and lung following pulmonary M.tb infection

\begin{tabular}{lcccccc}
\hline & \multicolumn{2}{c}{ BAL } & & & \multicolumn{2}{c}{ Lung } \\
\cline { 2 - 3 } \cline { 5 - 6 } & BCG & BCG/CF & & BCG & BCG/CF \\
\hline Day 0 & $77.4 \pm 30.8$ & $140.9 \pm 26.3$ & $1,792.6 \pm 499.3^{* *}$ & & $774 \pm 298$ & $1,180 \pm 396$ \\
Day 5 & $42.1 \pm 19.3$ & $1,590.1 \pm 442.4^{* * *}$ & & $3,123.3 \pm 110.9$ & $8,370 \pm 207.2$ \\
Day 10 & $1,512 \pm 615.7$ & $2045 \pm 357.5$ & & $6,443.3 \pm 1438$ & $9,316.6 \pm 4330.5$ \\
Day 14 & & & & & $4,834.5 \pm 1437.7$ & \\
\hline
\end{tabular}

$\mathrm{Ag}$, antigen; BAL, bronchoalveolar lavage; BCG, Bacillus Calmette-Guérin; CF, culture filtrate; IFN, interferon; M.tb, Mycobacterium tuberculosis.

Accelerated secondary airway luminal CD8 T cell responses to pulmonary M.tb $\mathrm{H}_{37}$ Ra challenge in parenteral BCG-immunized/CF-treated hosts. The absolute numbers of Ag-specific CD8 T cells in the BAL and lung interstitium analyzed at various time points post-M.tb challenge in the mice immunized with BCG alone (BCG) or immunized with BCG and treated with CF (BCG/CF). Data are expressed as mean values \pm s.e.m. of $4-5$ mice per group, representative of two independent experiments.

${ }^{\star} P<0.05,{ }^{* *} P<0.005,{ }^{* *} P<0.0005$ compared with BCG alone counterparts.

phases of M.tb infection (from 4 weeks onward) in BCG-immunized hosts is preceded by a marked response of ALT (day 14) (Figures $\mathbf{1 b}, \mathbf{d}$ and $\mathbf{6 b}, \mathbf{c})$. The fact that significantly increased protection lags behind markedly raised ALT suggests that ALTmediated protection takes some time to ramp up. In comparison, naïve non-immunized animals still lack ALT by day 14 after
M.tb challenge and, thus, do not have increased protection in the lung by 4 weeks. Also, we have observed a similarly delayed lung protection from M.tbH37Ra or $M . t b \mathrm{H} 37 \mathrm{Rv}$ challenge in BCG-immunized hosts, suggesting the delayed ALT responses to be a trait of infection by both attenuated and virulent strains of mycobacteria. These findings together suggest that although 
a

\section{Intratracheal transfer of T cells}
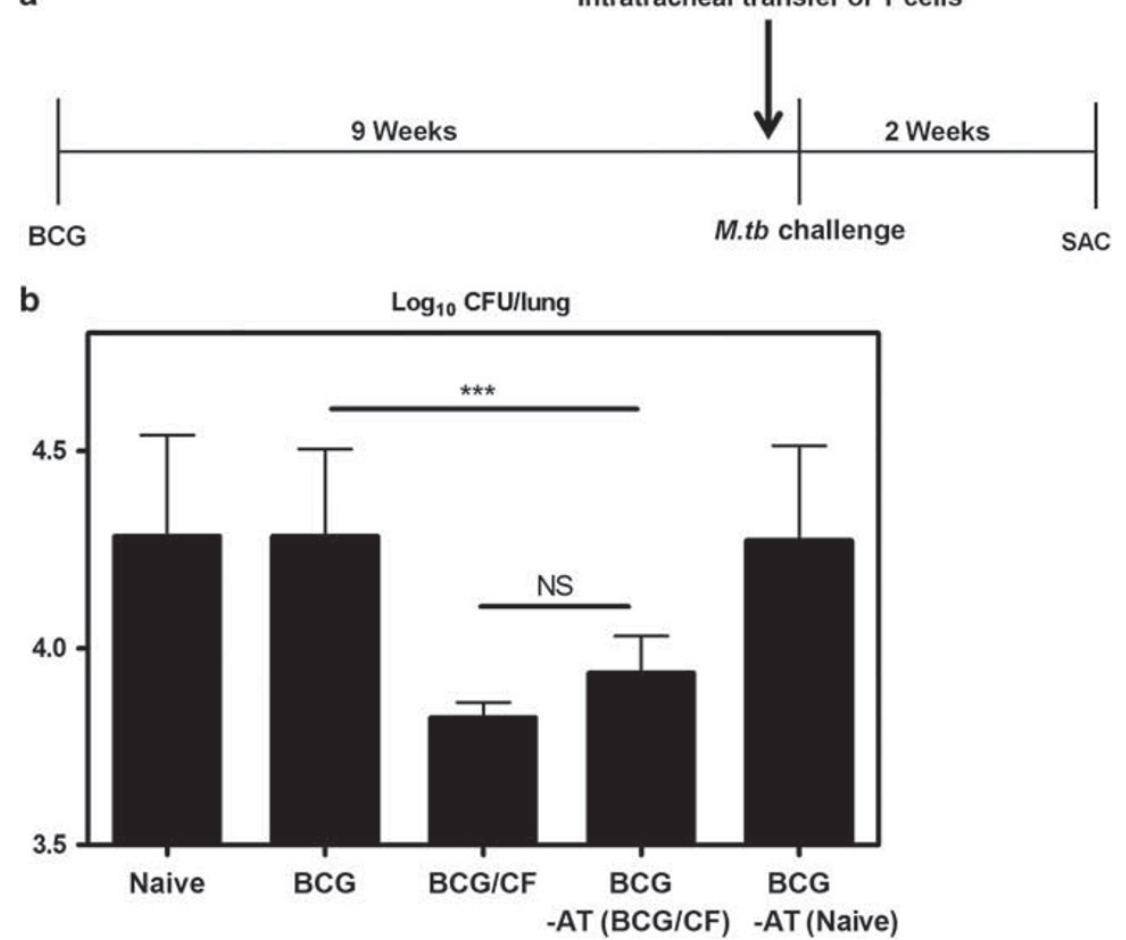

Figure 8 Adoptive transfer of antigen-specific T cells into the airway lumen of parenteral Bacillus Calmette-Guérin (BCG)-immunized mice restores lung protection in the early phase of pulmonary Mycobacterium tuberculosis (M.tb) infection. (a) Experimental schema. (b) The levels of immune protection in the lung (bacterial colony-forming units (CFU) burden) were assessed 2 weeks post- $M$.tb challenge in naïve mice, BCG-immunized mice, BCG-immunized/CF-treated mice (BCG/CF), BCG-immunized mice receiving the in vivo primed T cell transfer (BCG-AT (BCG/CF)), and BCG-immunized mice receiving the naive T-cell transfer (BCG-AT (naïve)) as control. Data are expressed as mean values \pm S.e.m. of five mice per group, representative of two independent experiments. ${ }^{* \star} P \leq 0.0005$ compared with the indicated groups. CF, M.tb culture-filtrate; NS, not statistically significant; SAC, sacrifice.

compared with naive non-immunized hosts, parenteral BCG immunization allows the primed $\mathrm{CD} 4 \mathrm{~T}$ cells to populate the lung interstitium, and upon pulmonary mycobacterial exposure, it accelerates T-cell recruitment to the airway lumen and lung interstitium by a few days, it still leaves a significant "immunological gap" where the lung is devoid of the ALT and left completely unprotected. We believe that this significant delay in engendering the ALT and lung protection in the early phases of pulmonary M.tb infection to be an important mechanism underlying the relative inefficacy of parenteral BCG immunization to protect from pulmonary TB in humans.

The molecular mechanisms responsible for the sluggish generation of ALT in response to pulmonary M.tb exposure in parenteral BCG-immunized hosts still remain to be fully understood. It, however, has been speculated that the slowgrowing nature of mycobacteria and the immune-evasive property of mycobacterial infection may have a role in delayed T-cell recruitment and lung protection. ${ }^{18}$ Regardless of which mechanisms, our current findings suggest that the future boosting strategies developed for enhancing protective efficacy of parenteral BCG immunization in humans should aim to fill up the "immunological gap" in the lung or to increase the number of Ag-specific T cells not only in the lung interstitium but also, more importantly, on the respiratory mucosal surface (airway lumen). As we have investigated in the current study, a potent way to accomplish this is to i.n. inoculate simple doses of crude non-formulated soluble mycobacterial proteins $(\mathrm{CF})$, which elicits a population of ALT that is highly expandable upon pulmonary M.tb exposure and helps accelerate lung protection in the early phases of pulmonary M.tb infection. Compared with the approach of respiratory mucosal boost vaccination, ${ }^{12,29}$ the airway delivery of CF differs in that it represents a "vaccineless" and perhaps even safer approach, as we have shown that the CF itself is incapable of T-cell priming and that it causes little undesired lung immunopathology. The potency of CF in eliciting and retaining the ALT in parenteral BCG-immunized animals is likely attributable to its capability to engage the TLRs and that it contains the mycobacterial antigens shared by BCG vaccine. Our previous studies suggest that both pro-inflammatory signals and mycobacterial Ags are required for eliciting and retaining the ALT. ${ }^{22,26}$

In summary, our current study has identified an immune mechanism, which we call an "immunological gap", underlying the delayed and unsatisfactory protection in the lung of parenteral BCG-immunized hosts. We believe that such knowledge will help develop the effective mucosal boosting strategies for enhancing the protective efficacy within the lung provided by parenteral BCG immunization in humans. 


\section{METHODS}

Mice. Female BALB/c mice aged 6-8 weeks were purchased from Harlan Laboratory (Indianapolis, IN). Mice were housed in a specific pathogen-free (level B) facility. All experiments were conducted in accordance with the animal research ethics board at McMaster University.

Mycobacteria. M. bovis BCG (Connaught), virulent M.tb. $\left(\mathrm{H}_{37} \mathrm{R}_{\mathrm{V}}\right.$; ATCC 27,294$)$ and attenuated $M . t b\left(\mathrm{H}_{37} \mathrm{Ra}\right.$; ATCC 25,177$)$ were grown in Middlebrook 7H9 broth supplemented with Middlebrook oleic acidalbumin-dextrose-catalase enrichment (Invitrogen Life Technologies, Carlsbad, CA), $0.002 \%$ glycerol, and $0.05 \%$ Tween 80 for $<10-15$ days, then aliquoted and stored in $-70^{\circ} \mathrm{C}$ until needed..$^{20,30,31}$ Before each use, $M . t b$ bacilli were washed with phosphate-buffered saline (PBS) containing $0.05 \%$ Tween 80 twice and passed through a 27 -gauge needle 10 times to disperse clumps.

BCG immunization and delivery of soluble M.tb antigenic proteins. Mice were immunized subcutaneously with $5 \times 10^{4}$ colony forming units (CFU) of BCG in $100 \mu \mathrm{l}$ of PBS. For experiments involving the administration of soluble M.tb CF proteins, recombinant Ag85A (rAg85A) or purified M.tb Ag85 complex proteins (Ag85 Complex containing purified Ag85A, B, and C proteins), $2 \mu \mathrm{g}$ of each in $20 \mu \mathrm{l}$ of PBS was delivered i.n. to BCG-immunized mice. All of these M.tb reagents were provided by the BEI Resources (Manassas, VA).

Pulmonary M.tb challenge. Mice were challenged i.n. with a dose of $0.5 \times 10^{6} \mathrm{CFU} M . t b \mathrm{H}_{37} \mathrm{Ra}$ in $40 \mu \mathrm{l}$ of PBS or with a dose of $1,000 \mathrm{CFU}$ (depositing 100-200 CFU to the lung) $M . t b \mathrm{H}_{37} \mathrm{Rv}$ in $25 \mu \mathrm{l}$ of PBS. The mice were killed at 2 or 4 weeks following M.tb challenge. The use of attenuated H37Ra strain allowed us to perform detailed analyses of airway luminal T-cell responses under the $\mathrm{P} 2$ conditions (we cannot perform the BAL in H37Rv-infected mice in the P3 facility due to technical constraint). The level of bacterial burden in the lung and spleen was assessed at each time point by plating serial dilutions of lung homogenates in triplicate onto plates containing Middlebrook 7H10 agar plates containing Middlebrook oleic acid-albumin-dextrose-catalase enrichment. Plates were placed into semisealed plastic bags and incubated at $37^{\circ} \mathrm{C}$ for 21 days. Colonies were then enumerated, calculated, and expressed as $\log _{10} \mathrm{CFU}$ per organ.

Lung histology. Isolated lungs were fixed for $72 \mathrm{~h}$ in $10 \%$ formalin, processed, paraffin embedded, sectioned, and stained with hematoxylin and eosin.

Cell isolation from the airway lumen, lung interstitium, and spleen. The intra-airway luminal cells were removed from the lung by exhaustive lavage as previously described. ${ }^{20,22}$ Following lavage, the lungs were perfused through the left ventricle with Hanks' buffer to remove leukocytes and red blood cells from the pulmonary vasculature. The lungs were then cut into small pieces and incubated with collagenase type 1 (Sigma-Aldrich, Oakville, Ontario, Canada) for $1 \mathrm{~h}$ at $37^{\circ} \mathrm{C}$. The digested lungs were then crushed through a $100-\mu \mathrm{m}$ filter. Splenocytes were isolated as previously described. ${ }^{20,22}$ All isolated cells were resuspended in RPMI 1640 medium supplemented with 5\% fetal bovine serum and $1 \%$ penicillin and streptomycin.

Immunological analyses by intracellular cytokine staining and fluorescence-activated cell sorting. Isolated mononuclear cells were cultured in a U-bottom 96 -well plate at a concentration of 20 million cells $\mathrm{ml}^{-1}$ for spleen and lung cells, and $0.5-1$ million cells $\mathrm{ml}^{-1}$ for cells from the BAL. For intracellular cytokine staining, cells were cultured for $24 \mathrm{~h}$ in the presence or absence of mycobacterial antigens (M.tb CF and crude BCG). Golgi plug (5 $\mathrm{g}$ g/brefeldin A; BD Pharmingen, Mississauga, Ontario, Canada) was added after $18 \mathrm{~h}$ of stimulation. Cells were then washed and blocked with CD16/CD32 in $0.5 \%$ bovine serum albumin/PBS for $15 \mathrm{~min}$ on ice and stained with cell surface antibodies.
Cells were washed, permeabilized, and stained with in accordance with the manufacturer's instructions for intracellular cytokine staining (BD Pharmingen). The following fluorochrome-labeled antibodies were used: CD3-Per chlorophyll protein Cy5.5, CD4-PE-Cy7, CD8-APC-Cy7, IFN$\gamma$-APC, TNF- $\alpha$-FITC, IL-2-PE, CD44-PE-Cy5, CD62 L-PE-Texas Red, CD127-FITC (BD Pharmingen). Following staining, cells were run on a LSR II (BD Pharmingen) and 100,000-200,000 events were collected for each sample. The fluorescence-activated cell sorting data were analyzed using FlowJo Software (Tree Star, Ashland, OR). The CD4 + T cells that were positive for interferon- $\gamma$ intracellular cytokine staining were defined as Ag-specific CD4+ T cells.

T-cell purification and adoptive airway luminal transfer. $\mathrm{T}$ cells were purified from the lung mononuclear cells isolated from naïve and BCG/ CF-treated mice by using Pan T Cell Isolation Kit II (Miltenyi biotec, Auburn, CA). Briefly, single-cell suspensions were then pooled and first incubated with a cocktail of biotin-conjugated monoclonal antibodies against CD11b, CD11c, CD19, CD45R, CD49b, CD105, Ter-119, and anti-major histocompatibility complex class II. Cells were then incubated with anti-biotin Microbeads (Miltenyi biotec) and passed through an MS column using the OctoMACS separator (Miltenyi biotec). The negative (unlabeled) fraction was collected and run through a second MS column to achieve highly purified T cells. T-cell purity was determined by fluorescence-activated cell sortingto be consistently $>90 \%$. Purified T cells were then adoptively transferred intratracheally $\left(2 \times 10^{6}\right.$ cells/mouse in $40 \mu \mathrm{l}$ PBS) into the airway lumen of recipient mice. Recipient mice were then challenged via the airway with $M . t b \mathrm{H}_{37} \mathrm{Rv} 16 \mathrm{~h}$ after adoptive transfer and killed 2 weeks post challenge for assessment of bacterial burden.

Statistical analysis. Statistical analysis was conducted to evaluate the significance between two differences. For two-sample comparison, Student's $t$-test was used. For comparison between two or more groups, analysis of variance was used; wherever applicable, a post hoc Fisher's least significant difference test was used for further comparison. The difference was considered to be statistically significant when $P<0.05$.

SUPPLEMENTARY MATERIAL is linked to the online version of the paper at http://www.nature.com/mi

\section{ACKNOWLEDGMENTS}

We thank BEI Resources for providing M.tb antigens. This work was supported by funds from the Canadian Institutes for Health Research.

\section{DISCLOSURE}

The authors declared no conflict of interest.

(C) 2012 Society for Mucosal Immunology

\section{REFERENCES}

1. WHO. Global tuberculosis control: a short update to the 2009 report. World Health Organization 48 (2009).

2. Aagaard, C., Dietrich, J., Doherty, M. \& Andersen, P. TB vaccines: current status and future perspectives. Immunol Cell Biol 87, 279-286 (2009).

3. Fletcher, $H$. \& McShane, $H$. Tuberculosis vaccines: current status and future prospects. Expert Opinion on Emerging Drugs 11, 207-215 (2006).

4. Trunz, B.B., Fine, P.E.M. \& Dye, C. Effect of BCG vaccination on childhood tuberculous meningitis and miliary tuberculosis worldwide: a metaanalysis and assessment of cost-effectiveness. The Lancet 367, 1173-1180 (2006)

5. Kaufmann, S.H., Hussey, G. \& Lambert, P.H. New vaccines for tuberculosis. Lancet 375, 2110-2119 (2010)

6. Romano, M. \& Huygen, $\mathrm{K}$. DNA vaccines against mycobacterial diseases. Expert Rev Vaccines 8, 1237-1250 (2009).

7. Xing, Z. \& Charters, T.J. Heterologous boost vaccines for bacillus Calmette-Guerin prime immunization against tuberculosis. Expert Rev Vaccines 6, 539-546 (2007).

8. Cooper, A.M. T cells in mycobacterial infection and disease. Curr Opin Immunol 21, 378-384 (2009). 
9. Belyakov, I.M. \& Ahlers, J.D. What role does the route of immunization play in the generation of protective immunity against mucosal pathogens? J Immunol 183, 6883-6892 (2009).

10. Holmgren, J. \& Czerkinsky, C. Mucosal immunity and vaccines. Nat Med 11, S45-53 (2005).

11. Triccas, J.A. \& Davenport, M.P. Infectious diseases: too little, too late for tuberculosis. Immunol Cell Biol 86, 293-294 (2008).

12. Jeyanathan, M., Heriazon, A. \& Xing, Z. Airway luminal T cells: a newcomer on the stage of TB vaccination strategies. Trends Immuno/ 31, 247-252 (2010).

13. Wolf, A.J. et al. Initiation of the adaptive immune response to Mycobacterium tuberculosis depends on antigen production in the local lymph node, not the lungs. J Exp Med 205, 105-115 (2008).

14. Russell, M.S., Iskandar, M., Mykytczuk, O.L., Nash, J.H.E., Krishnan, L. \& Sad, S. A reduced antigen load in vivo, rather than weak inflammation, causes a substantial delay in CD8+ T cell priming against Mycobacterium bovis (Bacillus Calmette-Guérin). J Immunol 179, 211-220 (2007).

15. Jung, Y.J., Ryan, L., LaCourse, R. \& North, R.J. Properties and protective value of the secondary versus primary Thelper type 1 response to airborne Mycobacterium tuberculosis infection in mice. J Exp Med 201, 1915-1924 (2005).

16. Khader, S.A. et al. IL-23 and IL-17 in the establishment of protective pulmonary CD4+ T cell responses after vaccination and during Mycobacterium tuberculosis challenge. Nat Immunol 8, 369-377 (2007).

17. Connor, L.M. et al. A key role for lung-resident memory lymphocytes in protective immune responses after BCG vaccination. Eur J Immuno/ 40, 2482-2492 (2010)

18. Cooper, A.M. Cell-mediated immune responses in tuberculosis. Annu Rev Immunol 27, 393-422 (2009).

19. Beverley, P.C. \& Tchilian, E.Z. Lessons for tuberculosis vaccines from respiratory virus infection. Expert Rev Vaccines 7, 1165-1172 (2008).

20. Santosuosso, M., Zhang, X., McCormick, S., Wang, J., Hitt, M. \& Xing, Z. Mechanisms of mucosal and parenteral tuberculosis vaccinations: adenoviral-based mucosal immunization preferentially elicits sustained accumulation of immune protective CD4 and CD8 T cells within the airway lumen. J Immunol 174, 7986-7994 (2005).

21. Ronan, E.O., Lee, L.N., Beverley, P.C.L. \& Tchilian, E.Z. Immunization of mice with a recombinant adenovirus vaccine inhibits the early growth of Mycobacterium tuberculosis after infection. PLOS ONE 4, e8235 (2009).

22. Santosuosso, M. et al. Mucosal luminal manipulation of T cell geography switches on protective efficacy by otherwise ineffective parenteral genetic immunization. J Immunol 178, 2387-2395 (2007).

23. Dietrich, J., Andersen, C., Rappuoli, R., Doherty, T.M., Jensen, C.G. \& Andersen, P. Mucosal administration of Ag85B-ESAT-6 protects against infection with Mycobacterium tuberculosis and boosts prior bacillus Calmette-Guerin immunity. J Immunol 177, 6353-6360 (2006).

24. Goonetilleke, N.P., McShane, H., Hannan, C.M., Anderson, R.J., Brookes, R.H. \& Hill, A.V.S. Enhanced immunogenicity and protective efficacy against Mycobacterium tuberculosis of Bacille CalmetteGuerin vaccine using mucosal administration and boosting with a recombinant modified vaccinia virus Ankara. J Immunol 171, 1602-1609 (2003).

25. Kohlmeier, J.E. \& Woodland, D.L. Memory T cell recruitment to the lung airways. Curr Opin Immunol 18, 357-362 (2006).

26. Jeyanathan, M. et al. Murine airway luminal antituberculosis memory CD8 T cells by mucosal immunization are maintained via antigen-driven in situ proliferation, independent of peripheral T cell recruitment. Am J Respir Crit Care Med 181, 862-872 (2010).

27. Begum, D. et al. Accelerated induction of mycobacterial antigen-specific CD8+ T cells in the Mycobacterium tuberculosis-infected lung by subcutaneous vaccination with Mycobacterium bovis bacille CalmetteGuérin. Immunology 128, 556-563 (2009).

28. Chen, L., Wang, J., Zganiacz, A. \& Xing, Z. Single intranasal mucosal Mycobacterium bovis BCG vaccination confers improved protection compared to subcutaneous vaccination against pulmonary tuberculosis. Infect Immun 72, 238-246 (2004).

29. Xing, Z. \& Lichty, B.D. Use of recombinant virus-vectored tuberculosis vaccines for respiratory mucosal immunization. Tuberculosis $\mathbf{8 6 , 2 1 1 - 2 1 7}$ (2006).

30. Wang, J., Santosuosso, M., Ngai, P., Zganiacz, A. \& Xing, Z. Activation of CD8 T cells by mycobacterial vaccination protects against pulmonary tuberculosis in the absence of CD4 T cells. J Immunol 173, 4590-4597 (2004).

31. Wang, J. et al. Single mucosal, but not parenteral, immunization with recombinant adenoviral-based vaccine provides potent protection from pulmonary tuberculosis. J Immunol 173, 6357-6365 (2004). 